\title{
Presumed monozygotic twins develop following transfer of an in vitro-produced equine embryo
}

\author{
Melissa Ann ROBERTS ${ }^{1}$, Kelly LONDON ${ }^{1}$, Lino Fernando GAMPOS-GHILLÓN ${ }^{1}$ * and \\ Joy Lynn ALTERMATT ${ }^{2}$ \\ ${ }^{1}$ Department of Animal Science, California Polytechnic State University, CA 93407, U.S.A. \\ ${ }^{2}$ Department of Dairy Science, California Polytechnic State University, CA 93407, U.S.A.
}

\begin{abstract}
An equine embryo produced by intracytoplasmic sperm injection (ICSI) was transcervically transferred to a recipient mare and pregnancy was confirmed via ultrasound examination on days 11, 12 and 15. On days 20 and 22, a single embryonic proper with a heartbeat was observed. On day 29, two embryos proper appeared during ultrasound examination, each possessing a heartbeat. Subsequent examinations on days 35 and 39 revealed continued viability and development of both embryos proper. On day 49, demise of both fetuses was present. Although no DNA analysis or post-partum examinations were performed, it is presumed that the fetuses were monozygotic twins based on membrane classification by ultrasound imaging as well as development occurring after the transfer of a single in vitro-produced embryo.
\end{abstract}

Keywords: equine, ICSI, twins

\author{
J. Equine Sci. \\ Vol. 26, No. 3 \\ pp. 89-94, 2015
}

Twinning is an unfavorable occurrence in the mare because of the high rate of abortion and poor post-natal development of foals that do survive to term [1]. Horses have developed natural mechanisms by which one dizygotic twin is reduced prior to the fetal stage if both embryos fixate in the same uterine horn [6]. This unilateral reduction occurs in $73 \%$ of pregnancies containing embryos that are the same size, and in $100 \%$ of pregnancies containing embryos that are of dissimilar size. It is postulated that this occurs by the deprivation of one embryo of embryonal-maternal exchange. Most dizygotic twin embryos that fixate bilaterally in the uterine horns enter the fetal stage of development, but are unlikely to survive to term [6]. Monozygotic twinning in the horse is rare in comparison to the occurrence of dizygotic twins.

There are a few different classifications of monozygotic twin pregnancies, as studied from human developmental biology [7]. The first, known as dichorionic diamniotic, is a result of embryo division prior to differentiation of the early embryonic cells. The second, monochorionic diamniotic

Received: February 26, 2015

Accepted: June 24, 2015

*Corresponding author. e-mail: 1camposc@calpoly.edu

(C)2015 Japanese Society of Equine Science

This is an open-access article distributed under the terms of the Creative Commons Attribution Non-Commercial No Derivatives (bync-nd) License $<$ http://creativecommons.org/licenses/by-nc-nd/3.0/ .
(MCDA), occurs if the inner cell mass divides during the blastocyst stage of development, prior to implantation. The third type is monochorionic monoamniotic, which results if division took place after implantation of the blastocyst. Of these, MCDA is the most common case reported in humans, accounting for $70-75 \%$ of all monozygotic twin pregnancies [7].

While mechanisms leading to the development of dizygotic twins are fairly well-established, relatively little is known about the factors that cause the development of monozygotic twins. In humans, it has been reported that there is an increased rate of monozygotic twinning after the use of assisted reproductive technologies (ART) [3]. Manipulation of the zona pellucida during procedures such as intracytoplasmic sperm injection (ICSI) or assisted hatching (AH) may play a role in this trend [3]. ICSI is used in humans as a treatment for male-factor infertility [14], and it is also used to produce equine embryos in vitro because standard in vitro fertilization has not yet been successful [8]. Other factors believed to have an effect on the monozygotic twinning rate following ART procedures include: the use of pro-ovulatory drugs, maternal age, temperature fluctuations, blastocyst transfer, and sub-optimal culture conditions [3, 14].

There have been previous reports of monozygotic twins resulting from the transfer of an in vivo-recovered embryo in the horse $[11,13]$. However, to our knowledge, there 
has only been brief mention of a prior case of monozygotic twins resulting from the transfer of a single equine blastocyst produced by ICSI [10]. In this report, the twins were aborted at day 76 and DNA analysis confirmed that they were monozygotic. We present another case in which a pair of presumed monozygotic twins arose from the transfer of an in vitro-produced equine embryo obtained through ICSI.

All experimental procedures using animals were approved by the Institutional Animal Care and Use Committee of California Polytechnic State University, San Luis Obispo.

A six year old Arabian mare was referred for assisted reproduction procedures involving follicular aspiration and ICSI. The stallion used for fertilization was deceased and a limited amount of semen remained stored. The mare had previously produced three live foals. One foal was obtained by artificial insemination, which she carried to term. The others were obtained by artificial insemination followed by embryo flush and transfer to recipient mares. Physical and breeding soundness examinations were performed upon the mare's arrival to university facilities and determined to be within normal limits according to the standards of the practicing veterinarians.

The mare underwent follicular aspiration of a preovulatory follicle as previously described [2]. Briefly, reproductive examinations were performed daily during estrus. The oocyte was collected by transvaginal aspiration of a $38 \mathrm{~mm}$ follicle $24 \mathrm{hr}$ after intramuscular (IM) administration of $1.5 \mathrm{mg}$ deslorelin (BET Labs, Lexington, KY, U.S.A.). Upon recovery, the oocyte was placed in maturation medium (Tissue Culture Medium 199 with Earle's salts supplemented with $10 \% \mathrm{v} / \mathrm{v}$ FCS) (Life Technologies, NY, U.S.A.) for approximately $18 \mathrm{hr}$ under $5 \% \mathrm{CO}_{2}$ in air at $38.5^{\circ} \mathrm{C}$ [4]. Frozen semen was used for sperm injections, as previously described with some modifications [2]. Briefly, motile spermatozoa were recovered after Percoll gradient centrifugation. A single spermatozoa was immobilized, captured, and injected into the ooplasm of a matured oocyte using a $5 \mu \mathrm{m}$ inner diameter needle and a micromanipulator (Fig. 1). The injected oocyte was cultured in Chemically Defined Medium (CDM) supplemented with $0.04 \%$ BSA for 7 days under $5 \% \mathrm{CO}_{2}, 5 \% \mathrm{O}_{2}$ and $90 \% \mathrm{~N}_{2}$ at $38.5^{\circ} \mathrm{C}$ [5].

The resulting day 7 embryo was trans-cervically transferred to a 4-year-old maiden paint-horse type mare three days after ovulation. This zona-enclosed blastocyst was graded 1.5 according to the 1998 International Embryo Transfer Society manual guidelines and contained 5\% extruded cells. The recipient had a body condition score of 5 (from the scale 1-9) at the time of transfer with a normal reproductive tract and no uterine cysts. The recipient was kept in a herd of mares, housed in an enclosed dry lot with free choice grass hay, salt/minerals, and no access to horses outside of the enclosure. The recipient mare was adminis-

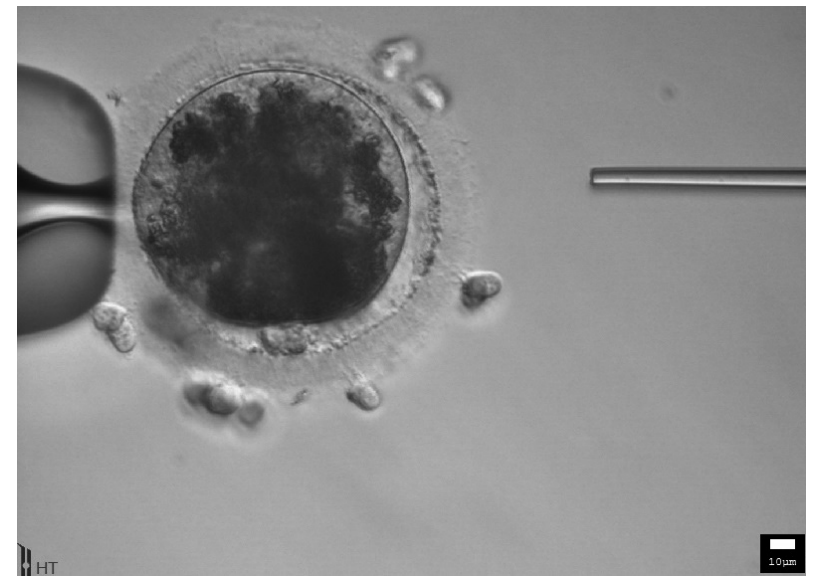

Fig. 1. Image showing the mature oocyte from the donor mare prepared for intracytoplasmic sperm injection (ICSI) with a single spermatozoa from the sire.

tered $600 \mathrm{mg}$ long-acting progesterone, IM prior to transfer along with $20 \mathrm{mg}$ acepromazine, IM and $500 \mathrm{mg}$ flunixin meglumine via intravenous (IV) injection (BET Labs).

Pregnancy determination was performed with ultrasound examination per rectum on days 11, 12 and 15 after ICSI and one embryonic vesicle was detected at each examination with respective measurements of 3, 6 and $18 \mathrm{~mm}$ (Fig. 2). On day 15 the corpus luteum of the recipient mare was also documented (Fig. 2). The entire uterus was scanned for the presence of a second vesicle at each examination and the second one was not detected. On days 20 and 22 after ICSI, one embryo proper measuring $2 \mathrm{~mm}$ on day 20 and $4 \mathrm{~mm}$ on day 22 was observed, with presence of a heartbeat on both days (Fig. 3). On day 29, two embryos proper were detected and the finding was documented (Fig. 4).

A more detailed examination of the uterus at day 29 revealed two viable embryos proper measuring 17 and $19 \mathrm{~mm}$, respectively, crown to rump (Fig. 5). Both embryos proper contained heartbeats, as examined via ultrasound color Doppler. The heartbeats are not able to be visualized from a still image simultaneously because they are located in different focal planes. A video image showing a transition through focal planes of the pregnancy demonstrates the presence of both heartbeats (Supplementary Video 1). The embryos proper were located in the center of the ultrasound image and membranes extended from both ends, connecting to the outer chorionic membrane. From this we can presume that there is a single external chorionic membrane and two separate allantoic and amniotic sacs without conjoining of the embryos proper, making these monozygotic, monochorionic, diallantoic, diamniotic twins.

At this stage in development, there were two options presented for the reduction of the twins. The first was to 


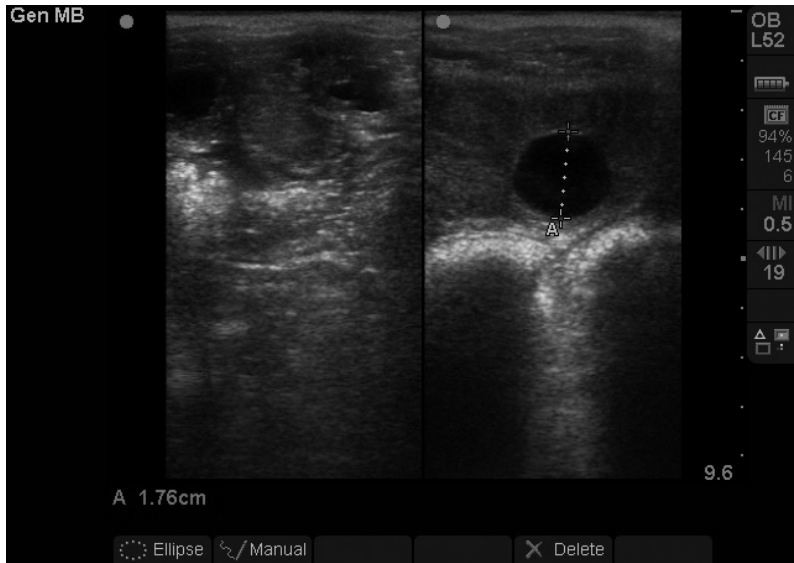

Fig. 2. Ultrasonographic images showing the corpus luteum of the recipient mare $(\mathrm{L})$ and the embryonic vesicle at day $15(\mathrm{R})$. Vesicle is approximately $18 \mathrm{~mm}$ in diameter.

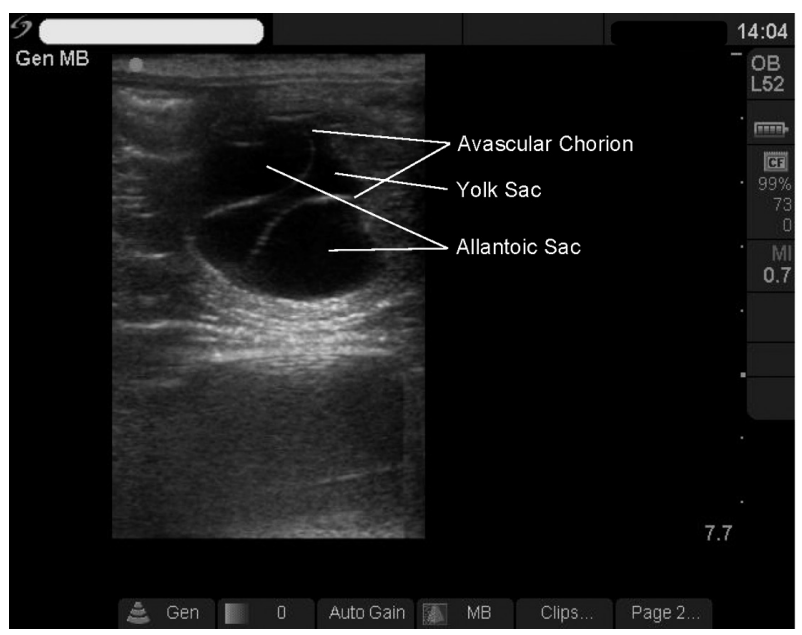

Fig. 4. Ultrasonographic image of the pregnancy at day 29 after ICSI. Labels show how membranes were classified upon examination.

perform ultrasound-guided transvaginal aspiration of one of the twins. The second option was to wait until 60 days gestation and perform cranio-cervical dislocation of one of the twins. It was decided by the owner of the donor Arabian mare that one twin would be reduced by cranio-cervical dislocation at day 60 of gestation. The recipient mare remained under long-acting progesterone supplementation by weekly IM injections throughout pregnancy monitoring.

On days 35 and 39, ultrasound examinations were performed and both twins were determined to be viable pregnancies (Figs. 6 and 7). On day 45, ultrasound examination demonstrated disruption of the fetal membranes characterized by an abnormal contouring of the membrane surfaces, indicating a loss of integrity of the membranes.

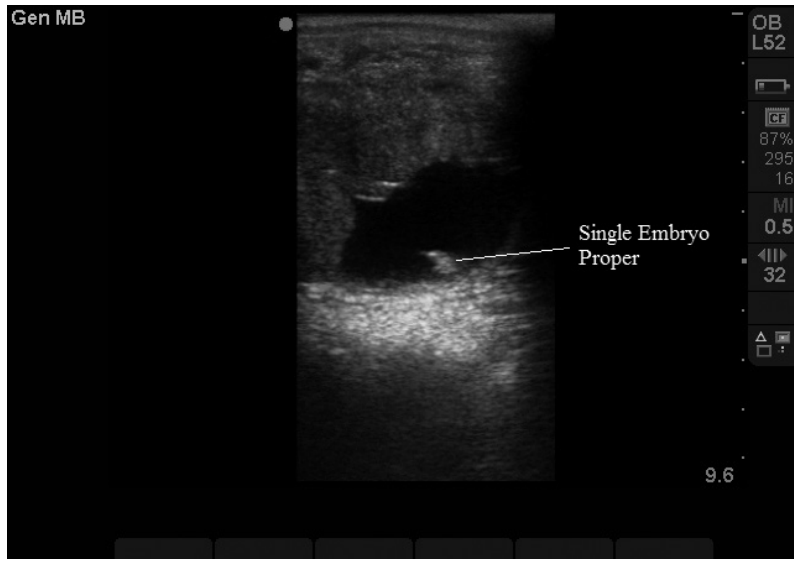

Fig. 3. Ultrasonographic image of pregnancy at day 22 showing a single embryo proper in the bottom right corner of the embryonic vesicle.

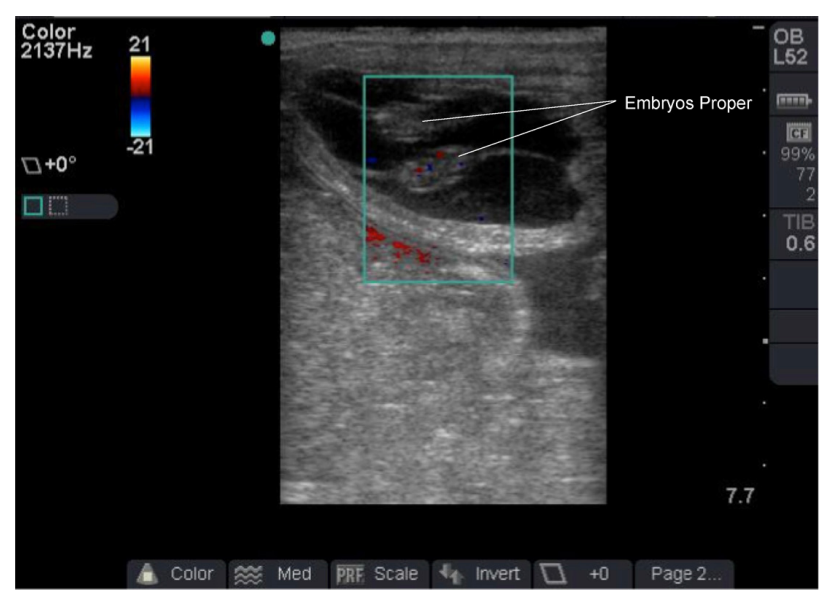

Fig. 5. Ultrasonographic image of the presumed monozygotic twins at day 29 after ICSI. Color Doppler demonstrates blood flow in the form of a heartbeat present in one embryo proper.

This disruption was accompanied by slight echogenicity of the allantoic fluid, however, heartbeats were still detected on both embryos proper (Fig. 8A). On day 49, echogenicity of the allantoic fluid increased and there was evidence of fetal demise as no observable heartbeat remained in either of the embryos proper (Fig. 8B).

The development of monozygotic twins from a single in vitro-produced equine embryo is a rare occurrence that illustrates the need for further studies regarding embryonic development following the use of ART in the horse. While the cause of fetal demise in this case is unknown, it is postulated that the spontaneous death of both twins after 45 days occurred due to compromised access to blood nutrients as a result of a shared chorioallantois and inadequacy of the placenta to support continued growth of both fetuses [6]. 


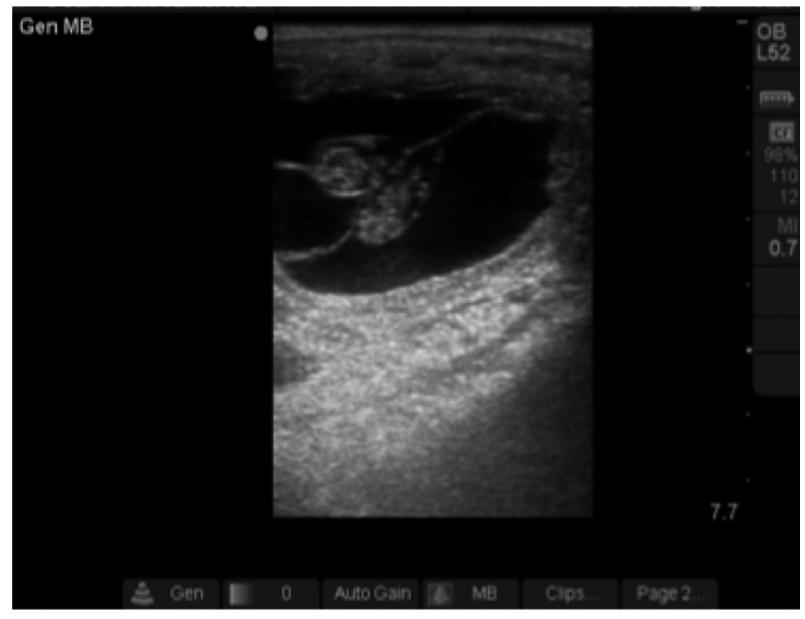

Fig. 6. Ultrasonographic image of the presumed monozygotic twins at day 35 after ICSI.
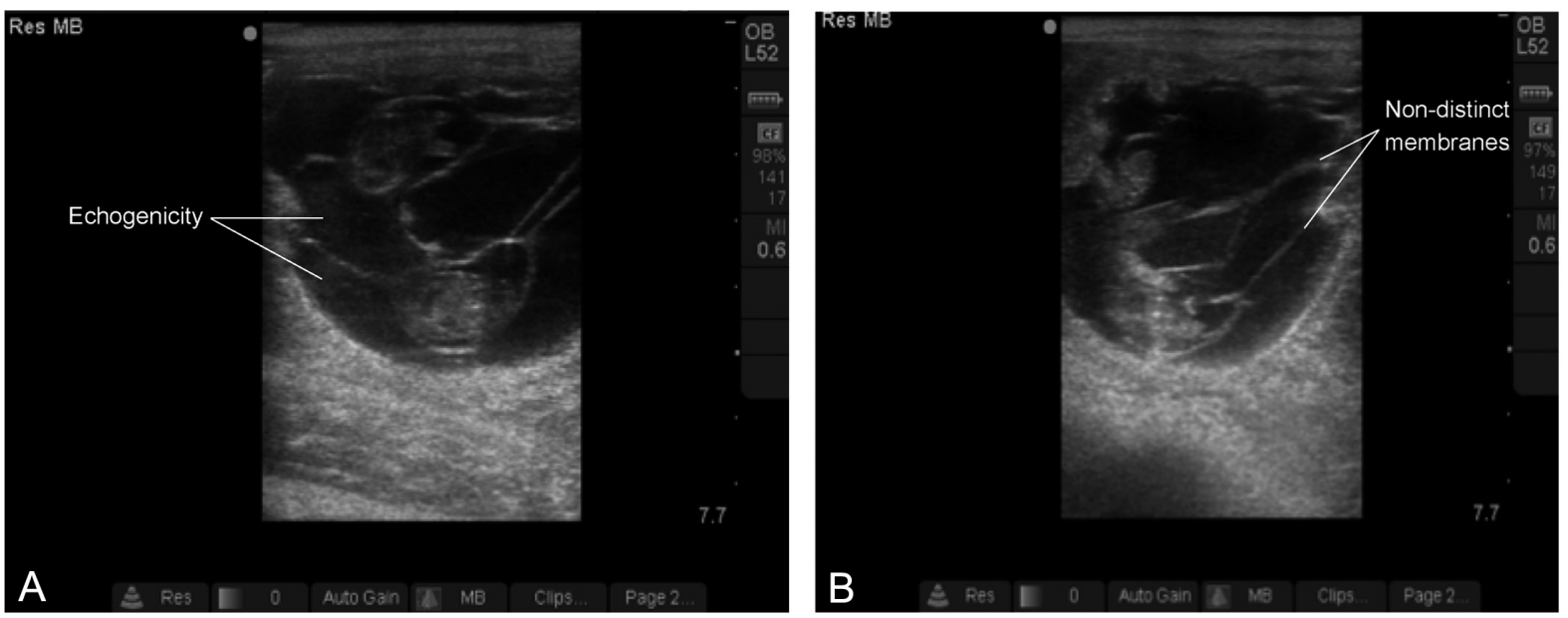

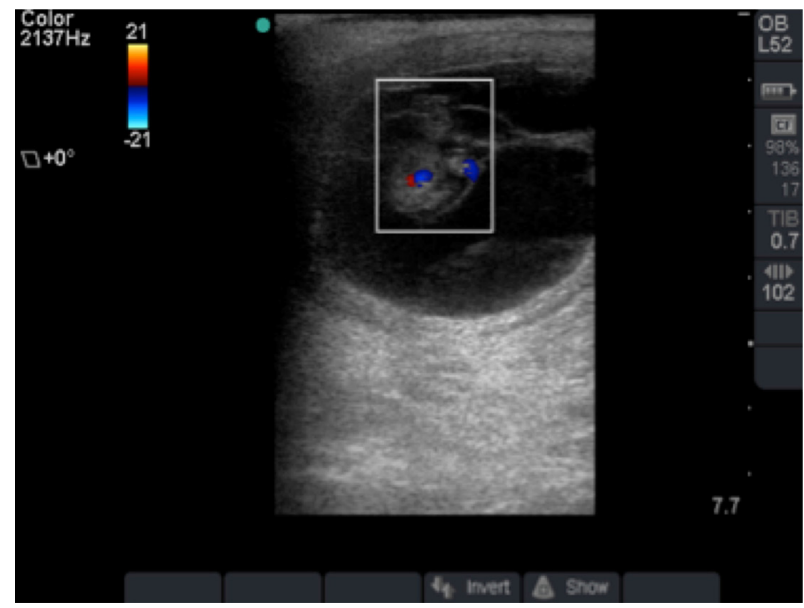

Fig. 7. Ultrasonographic image of the presumed monozygotic twins at day 39 after ICSI. Color Doppler demonstrates blood flow in the form of a heartbeat present in one embryo proper.

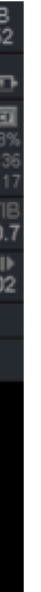

Fig. 8. Ultrasonographic images of the presumed monozygotic twins at days 45 and 49 after ICSI, respectively, showing disruption of fetal membranes, echogenicity of the allantoic fluid, and loss of fetal heartbeats.

Unilateral fixation of dizygotic twins typically results in the natural reduction of one of the embryos via deprivation of embryonal-maternal exchange and subsequent death of one embryo accompanied by survival of the embryo with greater vascularization [6]. In this case, however, fetal growth of two embryos proper developed after the transfer of a single embryo. These fetuses shared one external chorionic membrane and contained two separate allantoic and amniotic sacs (as visualized by ultrasound examination), making it unlikely that the twins were dizygotic and highly likely that they were monozygotic.

Multiple treatments have been developed to improve the outcome of twin pregnancies in the mare. However, most treatments apply to dizygotic twins in separate embry- 
chosen because this procedure has a higher success rate than transabdominal ultrasound-guided cardiac puncture and is less invasive [13].

Interestingly, we were unable to detect the second embryonic proper on days 20 and 22 when development of the allantoic sac begins and the embryonic proper is clearly visible. This may be attributed to the majority of the examinations being focused on the ventral portion of the uterus where the embryonic proper is normally oriented in the beginning stages of development. It is possible that a second embryonic proper was present but undetectable by ultrasound at that point if the ventral embryonic proper was larger than the dorsal one. Between days 24 and 36, the embryonic proper normally migrates towards the dorsal side of the uterus as the allantoic sac continues to develop and the yolk sac regresses, which is when the second embryonic proper was detected in this case [6]. Due to the type of membranes observed in this set of twins, it is presumed that the transfer of a portion of inner cell mass (ICM) cells within the blastocyst occurred after embryonic cell differentiation and before implantation [7]. It is possible that this ICM splitting occurred while the embryo was still in culture. Even though we monitored embryo development every 48 $\mathrm{hr}$ and prior to transfer, we were not able to detect a second ICM. Equine in vitro-produced embryos have an indistinct ICM at the blastocyst stage, which would make a detection such as this difficult [9].

Research on the causes of monozygotic twinning in the horse following the use of ART is limited. We therefore use the human as a model for postulation of possible causes of this apparent ICM splitting post-transfer. It has been shown through population analysis that ART significantly increases the occurrence of monozygotic twinning in humans if ICSI or AH is used, as well as if an embryo is transferred at day 5 rather than day 3 [12]. Manipulation of the zona pellucida is believed to cause a hole through which cells may herniate and then be pinched to create a second ICM or even an entirely new embryo. Day 5 human embryos have a higher survival rate after transfer than day 3 embryos, but it has been proposed that transferring later stage embryos may cause an increased risk of monozygotic twinning for reasons that have yet to be elucidated [12].

There are also multiple techniques believed to cause hardening of the zona pellucida, namely the use of ovulation-inducing hormones, as well as in vitro culture conditions [14]. In humans, it is believed that manipulation and hardening of the zona pellucida increase the rate of monozygotic twinning by complicating the hatching process and providing an opportunity for more compression than usual, at which point some of the ICM cells may be transferred to another location on the trophoblast outer layer [3]. This occurrence is unlikely in the equine embryo due to the presence of the unique embryonic capsule at day 6.5-7 of development and no true "hatching" event [6]. The equine embryo instead expands rapidly, thinning the zona pellucida until it essentially sheds or peels off. This leaves no single compression event required for the equine embryo to continue developing [6]. The caveat to this process, however, is that the embryonic capsule does not form in vitro. The blastocyst is transferred to a recipient mare while still enclosed in the zona pellucida and the capsule forms in vivo after transfer, at which point the zona pellucida sheds.

A possible cause of later embryonic splitting could be uterine contractions occurring during the mobile phase prior to fixation [6]. The capsule-enclosed equine embryo is a dynamic vesicle, able to withstand the movement incurred within the uterus. Uterine contractions could cause compression of the embryo to the point of transplanting cells from the ICM to the opposite side of the trophoblast cells, allowing for the development of a second embryo within the same chorionic membrane. However, because no significant research has been published on the causes of development of monozygotic twins following embryo transfer in the horse, we are unable to make definitive conclusions on this theory.

Of the factors that have been thought to cause monozygotic twinning in humans, suboptimal culture conditions, blastocyst transfer, and selective apoptosis seem to be the most applicable to the process of ICSI-produced embryo development in the horse [3]. While the in vitro culture conditions used to create equine ICSI embryos are the best we are able to produce for now, it would be incorrect to assume that they are without flaw. Mimicking the natural environment has yet to become a perfected process, making sub-optimal culture conditions a plausible cause of abnormalities observed in vitro-produced embryos. These conditions could have caused selective apoptosis of ICM cells, which may lead to a slight division in the ICM as a whole [3, 12]. These dead cells may have gone unnoticed prior to transfer and completed splitting after transfer. Also possible, the selective apoptosis could have occurred when the environment of the embryo was changed from an in vitro to an in vivo one.

Due to the lack of genetic analysis of the aborted twins, it can only be presumed that they were monozygotic based on ultrasound examination of the placenta and membranes. Ideally, a histopathological examination of the membranes as well as a DNA analysis of a non-hematopoietic tissue sample from each fetus would be needed to conclude that the two fetuses were indeed monozygotic [7]. Nevertheless, this is a rare occurrence and more research needs to be done on the causes of monozygotic twinning in the mare following the use of ART. 


\section{References}

1. Allen, W.R., and Pashen, R.L. 1984. Production of monozygotic (identical) horse twins by embryo micromanipulation. J. Reprod. Fertil. 71: 607-613. [Medline] [CrossRef]

2. Altermatt, J.L., Suh, T.K., Stokes, J.E., and Carnevale, E.M. 2009. Effects of age and equine follicle-stimulating hormone $(\mathrm{eFSH})$ on collection and viability of equine oocytes assessed by morphology and developmental competency after intracytoplasmic sperm injection (ICSI). Reprod. Fertil. Dev. 21: 615-623. [Medline] [CrossRef]

3. Aston, K.I., Peterson, C.M., and Carrell, D.T. 2008. Monozygotic twinning associated with assisted reproductive technologies: a review. Reproduction 136: 377-386. [Medline] [CrossRef]

4. Campos-Chillòn, L.F., Suh, T.K., Barcelo-Fimbres, M., Seidel, G.E. Jr., and Carnevale, E.M. 2009. Vitrification of early-stage bovine and equine embryos. Theriogenology 71: 349-354. [Medline] [CrossRef]

5. Campos-Chillòn, L.F., Walker, D.J., de la Torre-Sanchez, J.F., and Seidel, G.E. Jr. 2006. In vitro assessment of a direct transfer vitrification procedure for bovine embryos. Theriogenology 65: 1200-1214. [Medline] [CrossRef]

6. Ginther, O.J. 1998. Equine pregnancy: physical interactions between the uterus and conceptus, in Proceedings. Annual Convention of the AAEP. 44: 73-105.

7. Hall, J.G. 2003. Twinning. Lancet 362: 735-743. [Medline] [CrossRef]

8. Hinrichs, K. 2012. Assisted reproduction techniques in the horse. Reprod. Fertil. Dev. 25: 80-93. [Medline] [CrossRef]

9. Hinrichs, K., Choi, Y.H., Walckenaer, B.E., Varner, D.D., and Hartman, D.L. 2007. In vitro-produced equine embryos: production of foals after transfer, assessment by differential staining and effect of medium calcium concentrations during culture. Theriogenology 68: 521-529. [Medline] [CrossRef]

10. Li, X., Morris, L.H., and Allen, W.R. 2001. Influence of co-culture during maturation on the developmental potential of equine oocytes fertilized by intracytoplasmic sperm injection (ICSI). Reproduction 121: 925-932. [Medline] [CrossRef]

11. Mancill, S.S., Blodgett, G., Arnott, R.J., Alvarenga, M., Love, C.C., and Hinrichs, K. 2011. Description and genetic analysis of three sets of monozygotic twins resulting from transfers of single embryos to recipient mares. J. Am. Vet. Med. Assoc. 238: 1040-1043. [Medline] [CrossRef]

12. Skiadas, C.C., Missmer, S.A., Benson, C.B., Gee, R.E., and Racowsky, C. 2008. Risk factors associated with pregnancies containing a monochorionic pair following assisted reproductive technologies. Hum. Reprod. 23: 1366-1371. [Medline] [CrossRef]

13. Sper, R.B., Whitacre, M.D., Bailey, C.S., Schramme, A.J., Orellana, D.G., Ast, C.K., and Vasgaard, J.M. 2012. Successful reduction of a monozygotic equine twin pregnancy via transabdominal ultrasound-guided cardiac puncture. Equine Vet. Educ. 24: 55-59.

14. Toledo, M.G. 2005. Is there increased monozygotic twinning after assisted reproductive technology? Aust. N. Z. J. Obstet. Gynaecol. 45: 360-364. [Medline] [CrossRef]

15. Wolfsdorf, K.E. 2006. Management of postfixation twins in mares. Vet. Clin. North Am. Equine Pract. 22: 713-725. [Medline] [CrossRef] 